\title{
Knowledge Management and Organizational Learning
}

\author{
William R. King \\ Katz Graduate School of Business, University of Pittsburgh \\ wking115@yahoo.com
}

For centuries, scientists, philosophers and intelligent laymen have been concerned about creating, acquiring, and communicating knowledge and improving the re-utilization of knowledge. However, it is only in the last 15-20 years or so that a distinct field called "knowledge management" (KM) has emerged.

$\mathrm{KM}$ is based on the premise that, just as human beings are unable to draw on the full potential of their brains, organizations are generally not able to fully utilize the knowledge that they possess. Through KM, organizations seek to acquire or create potentially useful knowledge and to make it available to those who can use it at a time and place that is appropriate for them to achieve maximum effective usage in order to positively influence organizational performance. It is generally believed that if an organization can increase its effective knowledge utilization by only a small percentage, great benefits will result.

Organizational learning (OL) is complementary to KM. An early view of OL was “...encoding inferences from history into routines that guide behavior” (Levitt and March, 1988, p. 319). So, OL has to do with embedding what has been learned into the fabric of the organization.

\section{The Basics of Knowledge Management and Organizational Learning}

To understand $\mathrm{KM}$ and $\mathrm{OL}$, one must understand knowledge, $\mathrm{KM}$ processes and goals and knowledge management systems (KMS).

\subsection{Knowledge}

Knowledge is often defined as a "justified personal belief." There are many taxonomies that specify various kinds of knowledge. The most fundamental distinction is between "tacit" and "explicit" knowledge. Tacit knowledge inhabits the minds of people and is (depending on one's interpretation of Polanyi's (1966) definition) either impossible, or difficult, to articulate. Most knowledge is initially tacit in nature; it is laboriously developed over a long period of time through trial and error, and it is underutilized because "the organization does not know what it knows" (O'Dell and Grayson, 1998, p. 154). Some knowledge is embedded in business processes, activities, and relationships that have been created over time through the implementation of a continuing series of improvements. 
Explicit knowledge exists in the form of words, sentences, documents, organized data, computer programs and in other explicit forms. If one accepts the useful "difficult-to-articulate" concept of tacit knowledge, a fundamental problem of KM is to explicate tacit knowledge and then to make it available for use by others.

One can also distinguish among "know what," "know how" and "know why" levels of knowledge.

"Know what," knowledge specifies what action to take when one is presented with a set of stimuli. For instance, a salesperson who has been trained to know which product is best suited for various situations has a "know-what" level of knowledge.

The next higher level of knowledge is "know-how" - i.e., knowing how to decide on an appropriate response to a stimulus. Such knowledge is required when the simple programmable relationships between stimuli and responses, which are the essence of "know-what" knowledge, are inadequate. This might be the case, for instance, when there is considerable "noise" in symptomatic information so that the direct link between symptoms and a medical diagnosis is uncertain. "Know how"-type knowledge permits a professional to determine which treatment or action is best, even in the presence of significant noise.

The highest level of knowledge is "know-why" knowledge. At this level, an individual has a deep understanding of causal relationships, interactive effects and the uncertainty levels associated with observed stimuli or symptoms. This will usually involve an understanding of underlying theory and/or a range of experience that includes many instances of anomalies, interaction effects, and exceptions to the norms and conventional wisdom of an area.

\subsection{Knowledge Management Processes and Goals}

Knowledge management is the planning, organizing, motivating, and controlling of people, processes and systems in the organization to ensure that its knowledge-related assets are improved and effectively employed. Knowledge-related assets include knowledge in the form of printed documents such as patents and manuals, knowledge stored in electronic repositories such as a "best-practices" database, employees' knowledge about the best way to do their jobs, knowledge that is held by teams who have been working on focused problems and knowledge that is embedded in the organization's products, processes and relationships.

The processes of KM involve knowledge acquisition, creation, refinement, storage, transfer, sharing, and utilization. The KM function in the organization operates these processes, develops methodologies and systems to support them, and motivates people to participate in them.

The goals of KM are the leveraging and improvement of the organization's knowledge assets to effectuate better knowledge practices, improved organizational behaviors, better decisions and improved organizational performance.

Although individuals certainly can personally perform each of the KM processes, KM is largely an organizational activity that focuses on what managers can do to enable KM's goals to be achieved, how they can motivate individuals to participate in achieving them and how they can create social processes that will facilitate KM success.

Social processes include communities of practice - self-organizing groups of people who share a common interest - and expert networks - networks that are established to allow those 
with less expertise to contact those with greater expertise. Such social processes are necessary because while knowledge initially exists in the mind of an individual, for KM to be successful, knowledge must usually be transmitted through social groups, teams and networks. Therefore, $\mathrm{KM}$ processes are quite people-intensive, and less technology-intensive than most people might believe, although a modern knowledge-enabled enterprise must support KM with appropriate information and communications technology (King, 2008).

\subsection{Knowledge Management Systems}

Knowledge management systems (KMS) are applications of the organization's computer-based communications and information systems (CIS) to support the various KM processes. They are typically not technologically distinct from the CIS, but involve databases, such as "lessons learned" repositories, and directories and networks, such as those designed to put organizational participants in contact with recognized experts in a variety of topic areas.

A significant difference between many knowledge management systems and the organization's CIS is that the KMS may be less automated in that they may require human activity in their operation. While information systems typically require that humans make choices in the design phase and then operate automatically, KMS sometimes involve human participation in the operation phase. For instance, when a sales database is designed, people must decide on its content and structure; in its operational phase, it works automatically. When a "lessons learned" knowledge repository is created, people must make all of the same design choices, but they must also participate in its operational phase since each knowledge unit that is submitted for inclusion is unique and must be assessed for its relevance and important.

\section{Organizational Learning}

There are various ways to conceptualize the relationship between knowledge management and organizational learning.

Easterby-Smith and Lyles (2003) consider OL to focus on the process, and KM to focus on the content, of the knowledge that an organization acquires, creates, processes and eventually uses.

Another way to conceptualize the relationship between the two areas is to view OL as the goal of KM. By motivating the creation, dissemination and application of knowledge, KM initiatives pay off by helping the organization embed knowledge into organizational processes so that it can continuously improve its practices and behaviors and pursue the achievement of its goals. From this perspective, organizational learning is one of the important ways in which the organization can sustainably improve its utilization of knowledge.

Indeed, Dixon (1994), in describing an "organizational learning cycle," suggested that "accumulated knowledge... is of less significance than the processes needed to continuously revise or create knowledge" (p. 6). These processes are closely related to the notion of "continuous improvement" through which an organization continuously identifies, implements and institutionalizes improvements. The improvements are embedded in the organization through routines 
that may be written policies, prescribed machine settings, quality control limits or "best practices" for dealing with frequently occurring circumstances.

\section{Knowledge Management in Organizations}

Figure 1 shows that KM processes directly improve organizational processes, such as innovation, collaborative decision-making, and individual and collective learning. These improved organizational processes produce intermediate outcomes such as better decisions, organizational behaviors, products, services and relationships. These, in turn, lead to improved organizational performance.

\subsection{The Knowledge Management Processes Cycle}

Figure 2 is a process cycle model of KM. Such cycle models provide a useful way to organize one's thinking about KM processes. There have been numerous KM processes cycle models that describe the relationships of the key processes of KM, ranging from Davenport and Prusak's (2000) 3-stage model ("Generate, Codify/Coordinate, Transfer") to Ward and Aurum's (2004) 7-stage ("Create, Acquire, Identify, Adapt, Organize, Distribute, Apply").

The process cycle model of Fig. 2 is particularly valuable in that it uses the generally accepted terminology of KM and makes use of alternative paths in order to make important distinctions. The various activities listed as bullet-points under some of the major phases are meant to be illustrative and not necessarily definitional.

The model of Fig. 2 shows that the initiation of the KM cycle involves either the creation or the acquisition of knowledge by an organization. Knowledge creation involves developing new knowledge or replacing existing knowledge with new content (Nonaka, 1994). The focus of this is usually on knowledge creation inside the boundary of the firm or in conjunction with partners.

The four bullet points under "Creation" refer to Nonaka's (1994) four modes of knowledge creation - socialization (the conversion of tacit knowledge to new tacit knowledge through social interactions and shared experiences), combination (creating new explicit knowledge by merging,

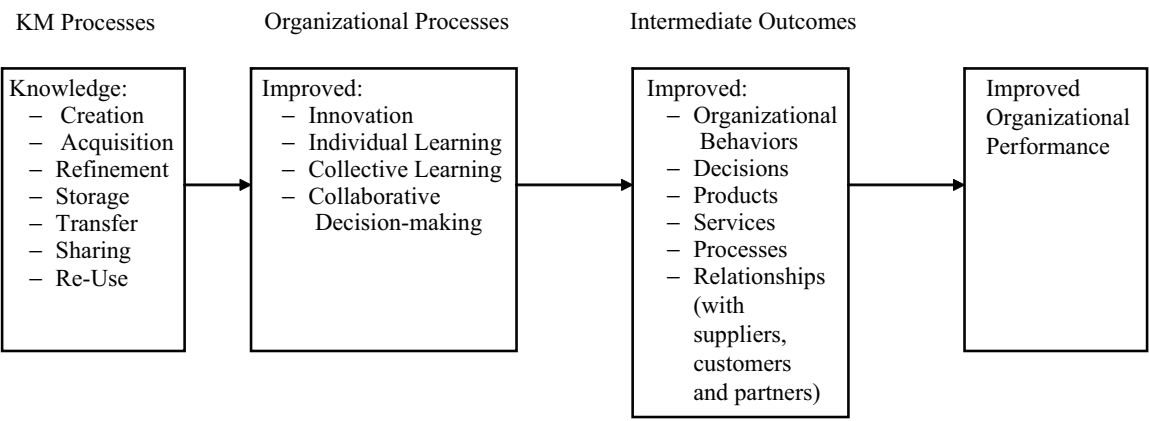

Fig. 1: KM in an Organization 


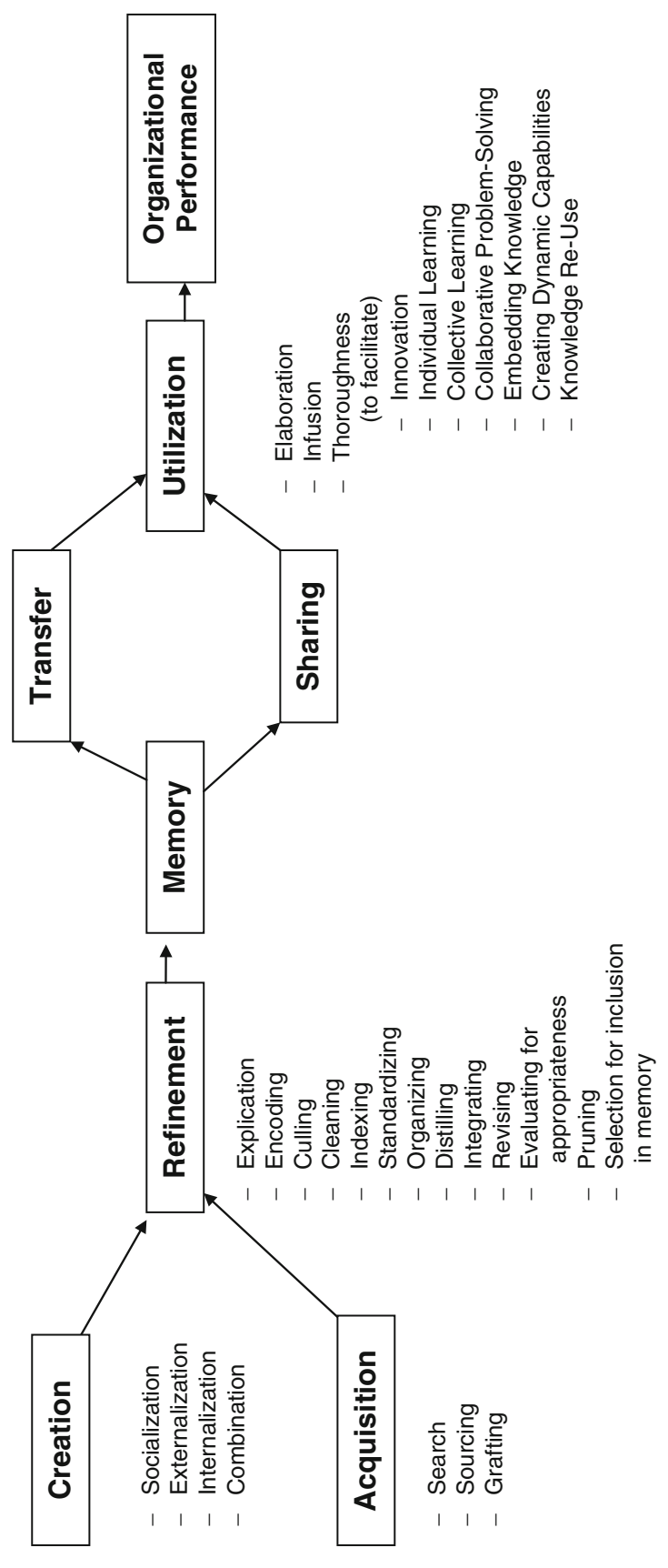

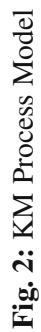


categorizing, and synthesizing existing explicit knowledge), externalization (converting tacit knowledge to new explicit knowledge) and internalization (the creation of new tacit knowledge from explicit knowledge). Illustrative of these four modes respectively are apprenticeships, literature survey reports, "lessons learned" repositories and individual or group learning through discussions.

In contrast to knowledge creation, knowledge acquisition involves the search for, recognition of, and assimilation of potentially valuable knowledge, often from outside the organization (Huber, 1991).

The bullet points under "Acquisition" illustrate some processes for acquiring knowledge from external sources - searching (as on the Internet) (Menon and Pfeffer, 2003), sourcing (selecting the source to use) (King and Lekse, 2006) and grafting (adding an individual who possesses desired knowledge to the organization) (Huber, 1991).

After new knowledge is created or acquired, KM mechanisms should be in place to prepare it to be entered into the organization's memory in a manner that maximizes its impact and longterm reusability. Knowledge refinement refers to the processes and mechanisms that are used to select, filter, purify and optimize knowledge for inclusion in various storage media.

Under "Refinement" in the figure, the bullet points suggest that tacit, or implicit, knowledge must be explicated, codified, organized into an appropriate format and evaluated according to a set of criteria for inclusion into the organization's formal memory. Of course, explicit knowledge needs only to be formatted, evaluated, and selected.

Of the various steps that are involved in doing so, "culling" refers to identifying the most significant exemplars in an emerging collection; "organizing" refers to identifying recurrent themes and linking individual knowledge items to the themes and "distilling" is creating a synopsis or set of pointers (McDonald and Ackerman, 1997).

Organizational memory includes knowledge stored in the minds of organizational participants, that held in electronic repositories, that which has been acquired and retained by groups or teams and that which is embedded in the business's processes, products or services and its relationships with customers, partners and suppliers(Cross and Baird, 2000).

As shown in the figure, in order for knowledge to have wide organizational impact, it usually must be either transferred or shared. Transfer and sharing may be conceptualized as two ends of a continuum. Transfer involves the focused and purposeful communication of knowledge from a sender to a known receiver (King, 2006a). Sharing is less-focused dissemination, such as through a repository, to people who are often unknown to the contributor (King, 2006b). Many of the points on the hypothetical continuum involve some combination of the two processes and both processes may involve individuals, groups or organizations as either senders or receivers, or both.

Once knowledge is transferred to, or shared with, others, it may be utilized through elaboration (the development of different interpretations), infusion (the identification of underlying issues), and thoroughness (the development of multiple understandings by different individuals or groups) (King and Ko, 2001) in order to be helpful in facilitating innovation, collective learning, individual learning, and/or collaborative problem solving (King, 2005). It may also be embedded in the practices, systems, products and relationships of the organization through the creation of knowledge-intensive organizational capabilities (Levitt and March, 1988). 
The end (right-side) of the cycle in Fig. 2 depicts knowledge having impact on organizational performance. Those who have an academic interest in KM sometimes forget that organizational performance improvement is what $\mathrm{KM}$ is ultimately all about. Anticipated improvements are the primary basis that organizations use to judge the value of KM initiatives. Many otherwiseworthy KM efforts are "shot down" because KM "experts" have not taken the effort to assess, forecast and adequately argue for their potential impact on the organization's goals of improved productivity, revenues, profits and return on investment.

\subsection{KM Strategies}

Most organizations focus primarily on one or the other of two broadly defined KM strategies "codification" or "personalization" (Hansen et al., 1999).

Codification, is primarily implemented in the form of electronic document systems that codify and store knowledge and permit its easy dissemination and re-use. This strategy is based on "re-use economics" - invest once in creating or acquiring a knowledge asset and re-use it many times.

Personalization, on the other hand, focuses on developing networks to facilitate people-topeople knowledge transfer and sharing. It is based on "expert economics" - channeling individual expertise to others with less expertise who may employ it to further the organization's goals.

Earl (2001) has described various KM strategies, or "schools of thought" at a more detailed level. He developed these empirically through observation in numerous companies. They are listed below in groups that emphasize their reliance on either the codification or a personalization approach.

Codification Sub-Strategies - Earl's codification-oriented sub-strategies are:

1. Systems (creating and refining knowledge repositories and on motivating people to provide content)

2. Process (developing and using repeatable processes that are supported with knowledge from previously conducted processes)

3. Commercial (the management of intellectual property such as patents, trademarks, etc.)

4. Strategic (the development of "knowledge capabilities" that can form the foundation of competitive strategy)

Personalization Sub-Strategies - Earl's personalization-oriented sub-strategies are:

5. Cartographic (creating knowledge "maps" or directories and networks to connect people)

6. Organizational (providing groupware and intranets to facilitate communities of practice)

7. Social (spatial) (socialization as a means of knowledge creation and exchange; emphasizes the providing of physical "places" to facilitate discussions)

While some organizations focus on only one of these strategies or sub-strategies, many use a combination of strategies that suits their needs.

\subsection{The Organization of KM}

$\mathrm{KM}$ is conducted in many different ways in organizations. Often, the KM function is headed by a Chief Knowledge Officer (CKO). If the organization's KM strategy is straightforward, the 
CKO may lead a KM Department. In more complex situations, with a diverse set of KM strategies being implemented, the cultural differences that are inherent in different strategies suggest that a single department may not be the best way to organize KM. In such instances, the communications linkages among various KM groups are of great importance (King, 2005; King, 2008)

Related to this is the perceived role of organizational culture in influencing KM practice and success. A "knowledge culture" is one particular variety of organizational culture representing a "way of organizational life that...enables and motivates people to create, share and utilize knowledge for the benefit and enduring success of the organization." (Oliver and Kandadi, 2006, p. 8). Organizational culture is believed to influence the knowledge-related behaviors of individuals, teams, organizational units and overall organizations because it importantly influences the determination of which knowledge it is appropriate to share, with whom and when.

\subsection{Extra-organizational KM}

KM may be conducted across multiple organizations, such as with suppliers, partners and customers. Such KM activities obviously rely on communications networks and systems (Van de Ven, 2005).

"Value supply chain" inter-organizational networks are in common usage to enable retailers such as Wal-Mart to interact with suppliers to ensure that inventories are always of desired levels on retail shelves, in retail stockrooms and in warehouses and that deliveries are made according to a predetermined schedule. These systems operate on an "automatic" basis that is made possible by the knowledge that is embedded in the software by the participating partners.

The well-known Linux software development project is an example of the effective utilization of a loose network of volunteer knowledge creators. It operates with two parallel structures - one which represents the current "approved" version of the system and the other in which enhancements are continuously being developed and tested (Lee and Cole, 2003).

\section{The Future of KM}

King et al. (2002) empirically identified a number of "KM issues" through a Delphi study of Chief Knowledge Officers. The resolution of these issues represents a forecast of how KM will be different in the future. The top 10 issues were:

- How to use KM to provide strategic advantage

- How to obtain top management support for KM

- How to maintain the currency of organizational knowledge

- How to motivate individuals to contribute their knowledge to a KM system

- How to identify the organizational knowledge that should be captured in KM systems

- How to assess the financial costs and benefits of KM

- How to verify the efficacy, legitimacy, and relevance of knowledge contributed to a KM system

- How best to design and develop a KM system 
- How to sustain progress in the organization

- How to ensure knowledge security

If all, or most, of these issues are resolved as KM matures, the future of KM will be largely determined by the manners in which they are resolved.

\section{Conclusion}

Knowledge management is a set of relatively new organizational activities that are aimed at improving knowledge, knowledge-related practices, organizational behaviors and decisions and organizational performance. KM focuses on knowledge processes - knowledge creation, acquisition, refinement, storage, transfer, sharing and utilization. These processes support organizational processes involving innovation, individual learning, collective learning and collaborative decisionmaking. The "intermediate outcomes" of KM are improved organizational behaviors, decisions, products, services, processes and relationships that enable the organization to improve its overall performance.

\subsection{Organization of the Volume}

This volume is organized into five sections.

After this introductory chapter authored by the volume editor, Sect. I, "Basic Concepts of Knowledge Management," provides up-to-date presentations of some of the fundamental ideas of the field. Frank Land's thoughtful essay, "Knowledge Management or the Management of Knowledge?", places KM in the long historical context of managing knowledge. The chapter by Kiku Jones and Lori Leonard, "From Tacit Knowledge to Organizational Knowledge for Successful KM," identifies organizational characteristics and KM initiative characteristics that may be antecedents or enablers of successful KM. The chapter by James Bloodgood, "Organizational Routines as Mechanisms for Knowledge Creation, Utilization and Storage," describes the role of routines in embedding knowledge into the organization and emphasizes that they may be difficult to manage. In the next chapter, David Schwartz and Doron Tauber present "A Maturity Model for Knowledge Management Systems Integration" which derives from an action research project that documented the development of $15 \mathrm{KM}$ and IS systems over a 5-year period.

Section II, which is titled "Knowledge Management Issues," begins with the chapter "Knowledge Diffusion in R\&D Groups: Re-examining the Role of the Technological Gatekeeper." In it, Eoin Whelan. Brian Donnellan and Willie Golden examine the traditional gatekeeper's role in the internet era and find that it has disappeared and been replaced by two new roles. In the next chapter, "Managing Asymmetries in Transferring Tacit Knowledge," Peter Sun discusses the behaviors that may occur in transferring tacit knowledge between two parties. Susanna Perez Lopez, Jose Manuel Montes Peon and Carmilo Jose Vazquez Ordas focus on "Information Technology as an Enabler of Knowledge Management: An Empirical Analysis" in the next chapter. The chapter by Richard Herschel and Ira Yermish deals with "Knowledge Management and Business Intelligence" and Line Gry Knudsen and Bo Bernard Nielsen treat "Antecedents of 
Procedural Governance in Strategic Alliances" in their chapter. The last chapter in this section by William Lekse deals with "Enterprise-Wide Management of Intellectual Property."

Section III dealing with "Knowledge Management Applications" begins with "Virtual Worlds as Platforms for Communities of Practice" by Lakshmi Goel, Iris Junglas and Blake Ives. “Open Innovation Through Online Communities" by Paul M. DiGangi and Molly Wasko discusses the incorporation of end-users into the organization's innovation process. Sajda Quershi, Mehruz Kamel and Peter Keen provide "Knowledge Networking to Overcome the Digital Divide" in the following chapter.

Section IV, "Measurement and Evaluation in KM and OL" begins with Meliha Handzic's "Evaluating KMS Effectiveness for Decision Support: A Preliminary Analysis." The next chapter is "Valuing Knowledge Within Virtual CoPs: The Quest for Meaningful Indicators" by PierreJean Barlatier, Yannick Naudet, Geraldine Vidou and Marie-Laure Watrinet. The chapter by Rene J Jorna, Niels Faber and Henk Hadders entitled "Organizational Knowledge, Cognitively Plausible Actors and Multi-Actor Systems" seeks to provide a basis for measuring organizational knowledge.

Section V treats "Organizational Learning." Chyan Yang and Liang-Chu Chen deal with the relationship between KM and OL in their chapter "On Using Organizational Knowledge Capabilities to Assist Organizational Learning." "Organizational Learning and Performance in Two National Cultures: A Multi-group Structural Equation Modeling Approach" by Miha Kerhvaj and VladDimovski empirically compares the impact of OL on organizational performance in two countries. The volume ends with Rene J. Jorna, Niels Faber and Henk Hadders' thoughtful essay titled "Sustainability, Learning, Adaptation and Knowledge Processing."

\section{References}

Cross, R., and L. Baird. (2000). "Technology is not enough: Improving performance by building organizational memory," Sloan Management Review, 41(3): 69-79.

Davenport, T.H., and L. Prusak. 2000. Working knowledge: How organizations manage what they know. Boston, MA: Harvard Business School Press.

Dixon, N.M. 1994. The organizational learning cycle: How we can learn collectively. New York: McGraw-Hill.

Earl, M. 2001. Knowledge management strategies. Journal of Management Information Systems 18(1): 215-223.

Easterby-Smith, M., and M. Lyles. 2003. The Blackwell handbook of organizational learning and knowledge management. Oxford: Blackwell.

Hansen, M.T., N. Nohria, and T. Tierney. 1999. What's your strategy for managing knowledge? Harvard Business Review 77(2): 106-116.

Huber, G.P. 1991. Organizational learning: The contributing processes and the literatures. Organization Science 2(1): 88-115.

King, W.R. 2005. Communications and information processing as a critical success factor in the effective knowledge organization. International Journal of Business Information Systems 10(5): 31-52.

King, W.R. 2006a. In "Knowledge sharing": The encyclopedia of knowledge management, D.G. Schwartz, 493-498. Hershey, PA: Idea Group Publishing. 
King, W.R. 2006b. In "Knowledge transfer": The encyclopedia of knowledge management, ed. D.G. Schwartz, 538-543. Hershey, PA: Idea Group Publishing.

King, W.R. 2008. An integrated architecture for the effective knowledge organization. Journal of Knowledge Management 12(2): 1367-1380.

King, W.R., and D.-G. Ko. 2001. Evaluating knowledge management and the learning organization: An information/knowledge value chain approach. Communications of the Association for Information Systems 5(14): 1-26.

King, W.R., and W. Lekse. 2006. Deriving managerial benefit from knowledge search: A paradigm shift? Information and Management 43(7): 874-883.

King, W.R., P. Marks, and S. McCoy. 2002. The most important issues in knowledge management. Communications of the ACM 45(9): 93-97.

Lee, G.K., and R.E. Cole. 2003. From a firm-based to a community-based model of knowledge creation: The case of the Linux Kernel development. Organization Science: A Journal of the Institute of Management Sciences 14(6): 633.

Levitt, B., and J.G. March. 1988. Organizational learning. Annual Review of Sociology 14: 319-340.

McDonald, D.W., and M.S. Ackerman. 1997. Collaborative refinery: A collaborative information workspace for the World Wide Web., Technical Report 97-03 Irvine: Information and Computer Science Department, University of California.

Menon, T., and J. Pfeffer. 2003. Valuing internal vs. external knowledge explaining the preference for outsiders. Management Science 49(4): 497.

Nonaka, I. 1994. A dynamic theory of organizational knowledge creation. Organizational Science 5(1): 14-37.

O’Dell, C., and C.J. Grayson. 1998. If only we knew what we know: identification and transfer of internal best practices. California Management Review 40(3): 154-174.

Oliver, S., and K.R. Kandadi. 2006. How to develop knowledge culture in organizations? A multiple case study of large distributed organizations. Journal of Knowledge Management 10(4): 6-24.

Polanyi, M. 1966. The tacit dimension. New York: Doubleday.

Van de Ven, A.H. 2005. Running in packs to develop knowledge-intensive technologies. MIS Quarterly 29(2): 365-378.

Ward, J., and A. Aurum. 2004. Knowledge management in software engineering - Describing the process, 137-146., 15th Australian Software Engineering Conference (ASWEC 2004) Melbourne, Australia: IEEE Computer Society Press. 